\title{
Safety of Nifedipine GITS in Stable Angina: The ACTION Trial
}

\author{
Philip A. Poole-Wilson', Bridget-Anne Kirwan', \\ Zoltán Vokó ${ }^{3}$, Sophie de Brouwer ${ }^{2}$, Fred J. van \\ Dalen $^{2}$, Jacobus Lubsen ${ }^{2,4}$, and on behalf of the \\ ACTION Investigators \\ ${ }^{1}$ Department of Cardiac Medicine, Imperial College, London, UK; \\ ${ }^{2}$ SOCAR Research, Nyon, Switzerland; ${ }^{3}$ The School of Public Health, \\ Medical and Health Science Centre, University of Debrecen, \\ Debrecen, Hungary; ${ }^{4}$ The Department of Epidemiology and \\ Biostatistics, Erasmus Medical Centre, Rotterdam, The Netherlands
}

Published online: 14 March 2006

\begin{abstract}
Summary. Aim: We describe the safety profile of nifedipine GITS as assessed from adverse events reported in the ACTION trial in which 7,665 patients with stable, symptomatic coronary artery disease were randomly assigned nifedipine GITS or placebo and followed for a mean of 4.9 years. Methods: All adverse events were coded using the COSTART coding dictionary. The incidence rate for each event was calculated as the number of patients with the event concerned divided by the total time 'at risk'. Hazard ratios comparing nifedipine with placebo and their $95 \%$ confidence intervals were obtained by Cox proportional-hazards analysis. Results: As reported previously, nifedipine significantly reduced the incidence of cardiovascular events and procedures [hazard ratio (HR) $0.89,95 \%$ confidence interval (CI) $0.83-0.95$ ]. Apart from the known side effects of nifedipine, which include peripheral oedema, vasodilatation, hypotension, asthenia, constipation, leg cramps, non-specific respiratory complaints, impotence and polyuria, and which were reported more frequently in patients assigned nifedipine, the incidence rates of most other adverse events were similar. There were no differences in the occurrence of gastrointestinal haemorrhage, myocardial infarction and suicide. The rate of occurrence of death or new cancer excluding non-melanoma skin cancer for patients with no history of cancer at baseline was 2.53/100 patient years for patients assigned nifedipine and $2.37 / 100$ patient years for patients assigned placebo (HR 1.06, 95\% CI 0.93-1.22). Conclusion: Overall nifedipine GITS was well tolerated by patients with stable symptomatic angina.
\end{abstract}

Key Words. randomised controlled trial, angina pectoris, nifedipine GITS, adverse events

\section{Introduction}

In the mid 1990s there was vigorous debate about the safety of calcium channel antagonists in the treatment of cardiovascular diseases [1-11]. A case-control study had suggested an increased risk of myocardial infarction in hypertensive patients treated with calcium channel antagonists [12]. Further questions about safety were raised in a meta-analysis of randomised, controlled trials in patients with ischaemic heart disease [13], and in the EPESE (Established Populations for Epidemiologic Studies of the Elderly) cohort study of antihypertensive medications in the elderly [14]. There was uncertainty whether the observed cardiovascular adverse effects were confined to short-acting formulations of calcium channel antagonists, or were a drug-class action [15]. In addition claims were made concerning an increased risk of cancer, major gastrointestinal haemorrhage, and suicide [16-21].

More recently, several studies with calcium channel antagonists have been reported mostly in patients with hypertension or with acute coronary syndromes. The findings refuted the earlier claims of harm [22-26]. However, data on the efficacy and safety of nifedipine, especially of the long-acting formulation, in stable angina was lacking. TIBET (Total Ischemic Burden European Trial) [27] showed no difference between slow-release nifedipine, atenolol and their combination for major cardiovascular endpoints in patients with stable angina while the withdrawal rate of study medication was highest in the combination group [28]. As TIBET was too small to assess effects on morbidity and mortality, the need for a large-scale and long-term trial remained. From the randomised, placebo-controlled ACTION trial (A Coronary disease Trial Investigating Outcome with Nifedipine GITS) in 7,665 patients with stable symptomatic coronary disease followed for a mean of 4.9 years, we have reported the effects of long-acting

The study was supported by Bayer Healthcare AG, Wuppertal, Germany.

Address for correspondence: Bridget-Anne Kirwan, SOCAR Research SA, PO Box 2564, 1260, Nyon 2, Switzerland. E-mail: bridget.kirwan@socar.ch 
nifedipine GITS (gastro-intestinal therapeutic system) on clinical outcomes [29]. The conclusion was that the addition of nifedipine GITS to the conventional treatment of angina pectoris was safe and survival free of a cardiovascular event or a procedure was prolonged. As is customary in drug trials, adverse events were recorded using the International Conference on Harmonisation (ICH) Good Clinical Practice (GCP) definitions. We report here the general safety profile of nifedipine GITS based on adverse events reported in the ACTION trial.

\section{Methods}

\section{Design}

The ACTION study was carried out in accordance with the provisions of the Declaration of Helsinki, ICH GCP requirements of the European Union and relevant local laws and regulations. Its design, methods and main results have been published previously [29, 30]. Briefly, patients aged 35 years or older with stable symptomatic angina pectoris requiring treatment were randomly assigned in equal proportions to either nifedipine GITS or matching placebo in addition to their current treatment. Apart from angina, patients had to have either a history of MI, proven angiographic coronary artery disease (CAD), or a positive exercise test or perfusion defect. The left-ventricular ejection fraction had to be at least $40 \%$. Major exclusions were: clinically significant heart failure, any major CV event or intervention within the last three months, planned coronary angiography or intervention, known intolerance to dihydropyridines, clinically significant valvular or pulmonary disease, unstable insulin-dependent diabetes mellitus, any gastro-intestinal condition that prohibited the use of GITS tablets, any condition other than CAD that limited life expectancy, hypotension or uncontrolled hypertension, and elevated creatinine or aminotransferase levels. Women could only participate if there was no risk of pregnancy. Detailed selection criteria and definitions have been described elsewhere [30].

Initial treatment was with nifedipine GITS $30 \mathrm{mg}$ once daily, increasing to $60 \mathrm{mg}$ once daily within six weeks, or matching placebo. Physicians were encouraged to attempt risk factor modification and to treat symptomatic angina with compatible medications. Lipid-lowering treatment was either continued or started at the same time as study medication according to internationally accepted guidelines. The following drugs could not be used in combination with study medication: calcium channel antagonists (2-week washout required before entry), cardiac glycosides (unless given for supra-ventricular arrhythmias), other positive inotropic agents, class I or III anti-arrhythmics (other than amiodarone or sotalol), cimetidine, anti-psychotic and anti-epileptic drugs, rifampicin or rifampine.

Patients were seen in the out-patient clinic two weeks, six weeks and six months after study medication was started, and from then onwards every six months. Between visits, patients were contacted by tele- phone. Reasons for stopping study medication earlier than planned were coded in the Case Report Form and included patient refusal, clinical reasons or contraindications, and the need to start concomitant treatment that was incompatible with study medication.

Non-fasting blood tests were performed prior to starting study medication for the following parameters: creatinine, total creatine kinase (CK), aspartate aminotransferase (ASAT), alanine aminotransferase (ALAT), total bilirubin, alkaline phosphatase, sodium, potassium, uric acid, glucose, total cholesterol, haemoglobin, haematocrit and white blood cell count. Thereafter, all tests except total cholesterol were repeated after 6 months, and after every two years. All tests including total cholesterol were repeated at the end of follow-up.

A 12-lead ECG was performed before starting study medication, and thereafter every 6 months. For each ECG the investigator recorded the ventricular rate (bpm), PQ, PR, QRS and QT intervals in milliseconds (ms) and ST-depression in millivolts $(\mathrm{mV})$, and coded rhythm, conduction, and wave morphology abnormalities.

\section{Adverse event reporting}

All patients were followed from the first intake of study medication onwards until death or the pre-defined end of follow-up irrespective of withdrawal of study medication beforehand. The sponsor's standard adverse event report forms based on CIOMS (Council for International Organizations of Medical Sciences) recommendations were used to document adverse events. An adverse event was defined as any undesirable experience that occurred during the trial, whether or not considered related to study medication. This included new laboratory test and ECG abnormalities considered clinically relevant by the investigator. An adverse event which was fatal, life threatening, disabling, resulted in or prolonged hospitalisation, or concerned the occurrence of cancer was considered as serious. Serious adverse events were reported within 24 hours of the investigator's awareness on the sponsor's standard two-page serious adverse event report form. The serious adverse event form was also used to report major diagnostic and intervention procedures irrespective of their indication. Based on the terms reported by the investigator, all adverse events were coded using the COSTART (coding symbols for thesaurus of adverse reaction terms) coding dictionary [31].

In our analysis, incidence of new cancer is only considered in patients without a baseline history of cancer. Pathological reports were obtained for all cases of new cancer during follow-up. The composite of death and incidence of any cancer is reported since patients may have died with undiagnosed cancer. Cancers excluding nonmelanoma skin cancers are reported since the diagnosis and ascertainment of skin cancer can be misleading. This approach has been used in major trials, [32, 33] one of which [33] was the trial upon which the power calculation for ACTION was based [30]. 


\section{Statistical methods}

We used the SLAIN (slope adjusted for intercept) method [34] to assess effects of nifedipine on the rate of change of laboratory parameters and QT, QRS, PQ/PR ECG intervals. The SLAIN analysis estimates the slope and intercept for each patient, using the laboratory test values measured at baseline and during follow-up as dependent, and time as an independent variable. The slopes are then correlated with study drug treatment while adjusting for intercept.

We considered all adverse events reported as of the first intake of study medication up to the end of followup for inclusion in the present report. For each adverse event, we calculated by assigned treatment the incidence rate, taken as the number of patients with the event concerned divided by the total time 'at risk'. For this purpose, we combined serious and non-serious adverse events, and ignored if the patient was using double-blind study medication at the time of the event or not. Hazard ratios (HRs) comparing nifedipine with placebo and their $95 \%$ confidence intervals (CIs) were obtained by Cox proportional-hazards analysis using treatment allocation as the only covariate. No adjustment for multiple testing was made.

Given the large number of reported cardiovascular and non-cardiovascular adverse events, we limited tabulation of events to the total that were reported for each COSTART body system. In addition, we grouped cardiovascular COSTART codes referring to the same clinical entity (see Appendix) and tabulated all cardiovascular adverse events reported in ACTION for which there was a statistically significant (95\% CI of HR does not include unity) difference between treatment groups, or that are mentioned in the manufacturer's core data sheet for nifedipine GITS, or which occurred at a rate of at least $0.5 / 100$ patient-years. For the noncardiovascular events, we tabulated for each body system class those events that occurred statistically significantly more frequently in one treatment group than in the other, or are mentioned in the core data sheet for nifedipine GITS as an untoward experience occurring in at least $1 \%$ of patients, or were malignant. In addition, we tabulated gastrointestinal bleeding (after combining events as indicated in the Appendix), depression and suicide attempt because these diagnoses have been suggested to be related to calcium channel antagonists [23]. Finally, we tabulated all events diagnosed as malignant separately and irrespective of their frequency.

\section{Results}

As reported elsewhere [29], ACTION was completed as planned. Study medication was started in 7,665 patients (3 825 nifedipine GITS, 3840 placebo). Key characteristics of the ACTION population are shown in Table 1.

Alkaline phosphatase showed an upward trend of $1.13 \mathrm{U} / \mathrm{L}$ per year of follow-up in patients allocated nifedipine as opposed to $0.02 \mathrm{U} / \mathrm{L}$ per year in patients assigned placebo ( $p=0.006$, SLAIN method). Bilirubinaemia and hyperuricaemia were reported more frequently in patients assigned placebo than patients assigned nifedipine (HR 0.35, 95\% CI 0.14-0.90; and HR $0.66,95 \%$ CI $0.48-0.91$ respectively, c.f. Figure 3$)$. There were no differences between treatment groups for the other laboratory tests performed.

There were no effects on the PQ, QRS and QT intervals in electrocardiograms. Effects of nifedipine on changes of heart rate and blood pressure have been published [29].

The total number of reported adverse events for patients assigned nifedipine and to placebo was similar (41 014 nifedipine, 40775 placebo), as was the number of patients who experienced at least one adverse event (96\% in both treatment groups). The percentage of patients who experienced at least one serious adverse event was similar in both treatment groups (58\%) but in total fewer serious adverse events were reported for patients assigned nifedipine (8 191 as opposed to 8530 for placebo). Study medication was withdrawn permanently two or more days before death or the end of follow-up in 1305 (34\%) patients assigned nifedipine and in 1179 (31\%) patients assigned placebo. The most frequent reason for stopping study medication was refusal to continue (11\% of patients assigned nifedipine vs. $10 \%$ of patients assigned placebo) or intolerable signs or symptoms that could not be controlled by dose reduction (11\% of patients assigned nifedipine vs. 5\% of patients assigned placebo, $p<0.001$ ).

Figure 1 shows adverse events using the COSTART body system classification. The incidence rates of adverse events within each COSTART body system were similar in both treatment groups except for body as a whole, cardiovascular, musculoskeletal, respiratory and urogenital. Cardiovascular events excluding peripheral oedema and respiratory events were significantly more frequent in patients assigned placebo; while body as a whole, cardiovascular events including peripheral oedema, musculoskeletal and urogenital events were significantly more frequent in patients assigned nifedipine.

Figure 2 shows a breakdown of adverse events within the COSTART body system cardiovascular. As expected, assignment to nifedipine was associated with a significantly increased incidence of peripheral oedema, hypotension and vasodilatation, and significantly reduced incidence of angina pectoris, hypertension, heart failure, bradycardia, and pericardial effusion.

Figures 3 and 4 show the occurrence of selected (c.f. statistical methods) events for each COSTART body system. For the COSTART body system body as a whole, asthenia and viral infection occurred more frequently in the nifedipine group. There was no difference in the rate of reported suicide attempts between the two treatment groups. For the COSTART body system digestive, constipation was significantly more frequent in patients assigned nifedipine whereas the rate of diarrhoea was significantly less in patients assigned nifedipine. There was no difference in the rate 


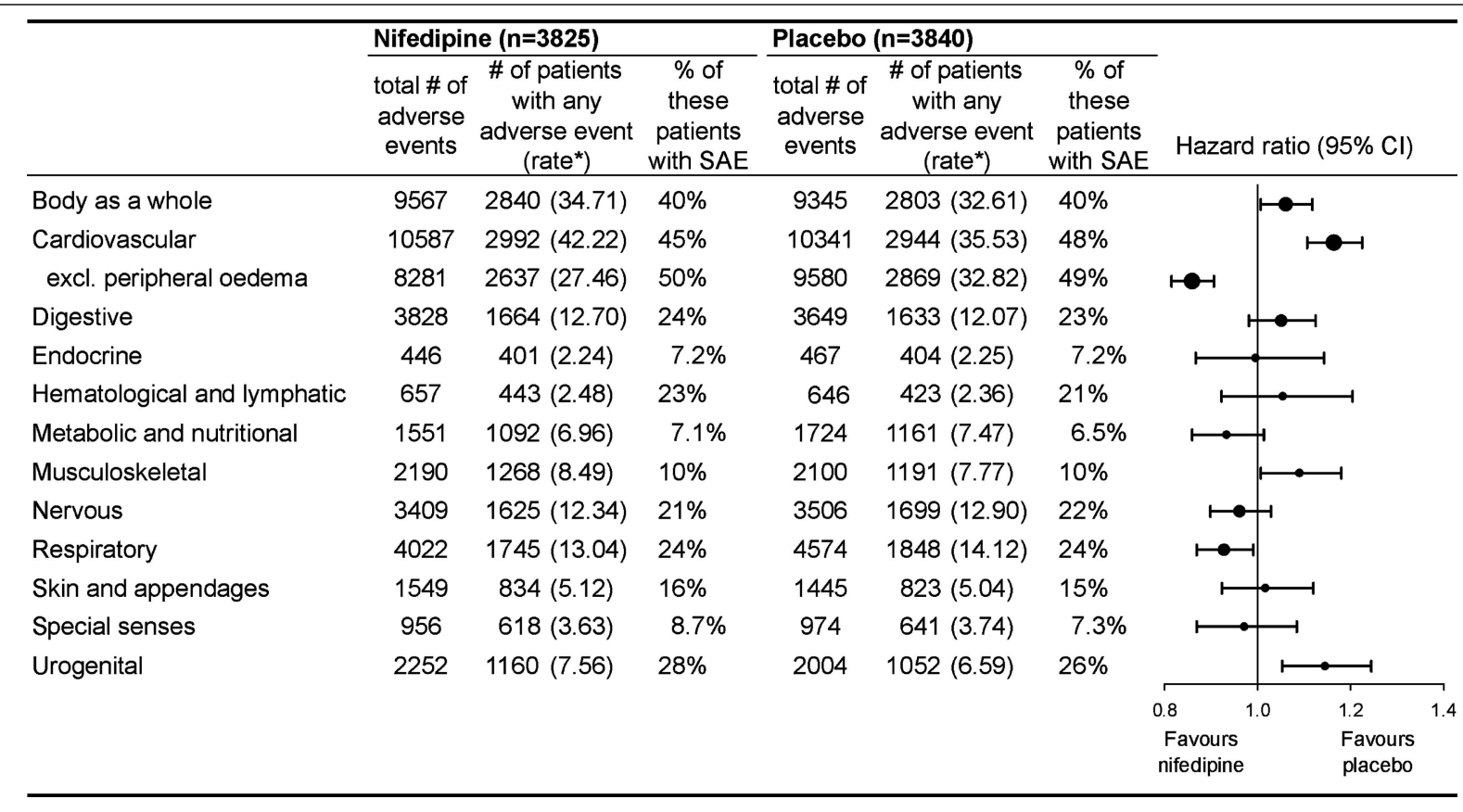

Fig. 1. Combined occurrence of adverse events by COSTART body system class. Note: Dot size indicating the hazard ratio corresponds to the percentage of patients concerned $<33 \%$ (smallest dot), 33-66\% (medium size dot), $>66 \%$ (largest size dot). SAE denotes serious adverse event and CI confidence interval. ${ }^{*}$ Number of patients with event per 100 patient-years at risk.

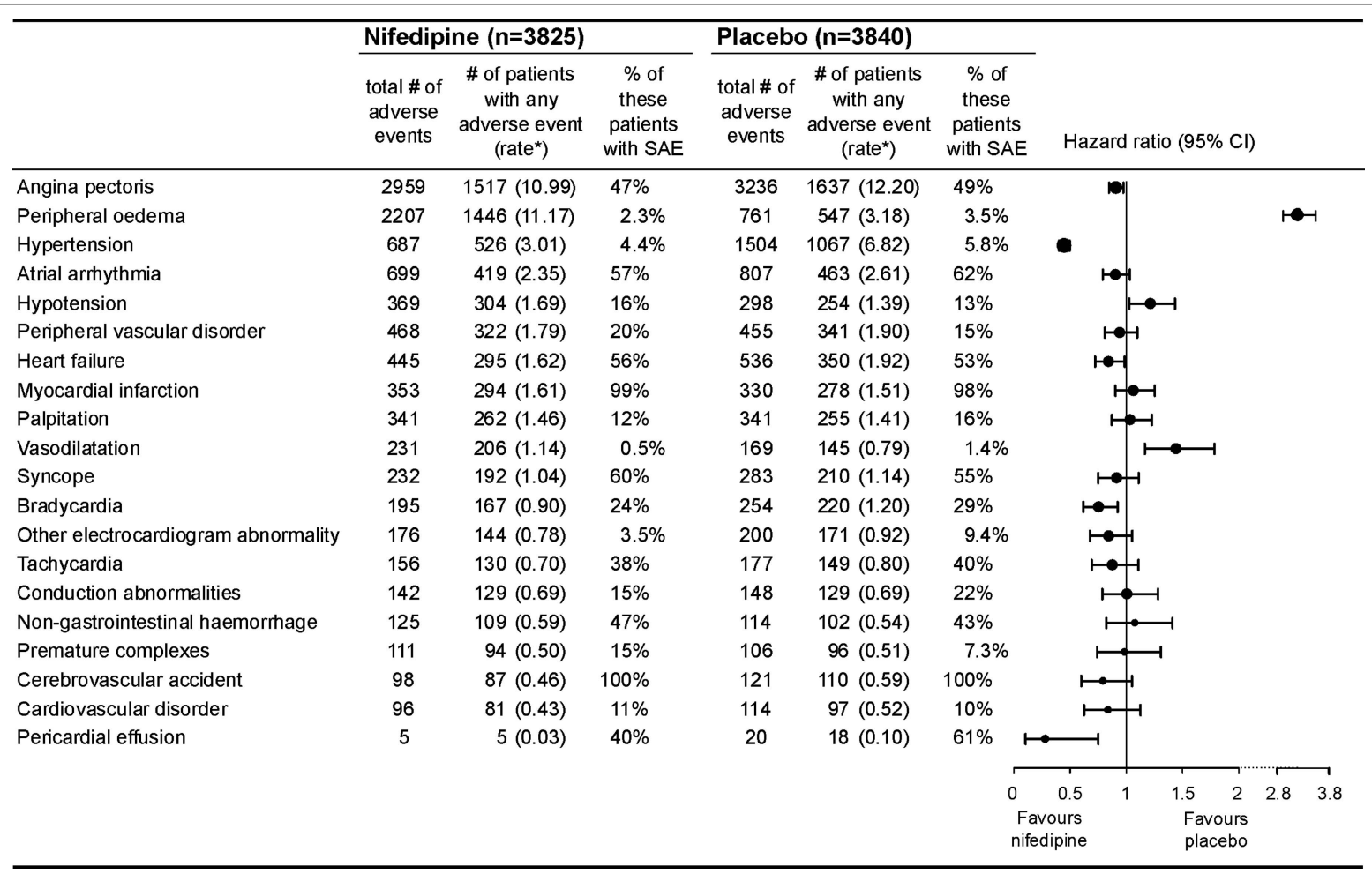

Fig. 2. Break-down of cardiovascular adverse events. Note: Dot size indicating the hazard ratio corresponds to the percentage of patients with the event concerned $<3 \%$ (smallest dot), $3-15 \%$ (medium-size dot), $>15 \%$ (largest size dot). SAE denotes serious adverse event and CI confidence interval. ${ }^{*}$ Number of patients with event per 100 patient-years at risk. 
Table 1. Key baseline characteristics of ACTION patients and main results

\begin{tabular}{|c|c|c|c|}
\hline & $\begin{array}{l}\text { Nifedipine } \\
(n=3825)\end{array}$ & & $\begin{array}{l}\text { Placebo } \\
(n=3840)\end{array}$ \\
\hline \multicolumn{4}{|l|}{ Baseline characteristics } \\
\hline Mean age (years) (SD) & $63.5(9 \cdot 3)$ & & $63.4(9.3)$ \\
\hline Male gender $(\%)$ & $3041(80)$ & & $3043(79)$ \\
\hline History of MI (\%) & $1974(52)$ & & $1924(50)$ \\
\hline Angiographic CAD, no MI (\%) & $1222(32)$ & & $1249(33)$ \\
\hline $\begin{array}{l}\text { Positive exercise or perfusion test, no history } \\
\text { of MI and angiography never done (\%) }\end{array}$ & $616(16)$ & & $646(17)$ \\
\hline Current NYHA class II-III (\%) & $1756(46)$ & & $1776(46)$ \\
\hline Anginal attacks (\%) & $3544(93)$ & & $3526(92)$ \\
\hline History of peripheral CV disease $(\%)^{\mathrm{a}}$ & $494(13)$ & & $491(13)$ \\
\hline Diabetes mellitus (\%) & $567(15)$ & & $546(14)$ \\
\hline \multicolumn{4}{|l|}{ Risk factors: } \\
\hline Current smoker (\%) & $686(18)$ & & $670(17)$ \\
\hline Total cholesterol $\geq 5.0 \mathrm{mmol} / \mathrm{l}(\%)$ & $2382(62)$ & & $2433(63)$ \\
\hline Body mass index $\geq 30.0(\%)$ & $849(22)$ & & $895(23)$ \\
\hline BP 140/90 mm Hg or higher (\%) & $1975(52)$ & & $2002(52)$ \\
\hline Any of the above (\%) & $3291(86)$ & & $3362(88)$ \\
\hline \multicolumn{4}{|l|}{ Patients with event during follow-up: } \\
\hline Death from any cause (rate $\left.{ }^{b}\right)$ & $310(1.64)$ & & $291(1.53)$ \\
\hline & & HR 1.07, 95\% CI 0.91-1.25 & \\
\hline Primary endpoint for safety ${ }^{c}\left(\right.$ rate $\left.^{b}\right)$ & $562(3.08)$ & HR $1.01,95 \%$ CI $0.90-1.14$ & $558(3.05)$ \\
\hline Primary endpoint for efficacy ${ }^{d}\left(\right.$ rate $\left.^{b}\right)$ & $804(4.60)$ & HR $0.97 .95 \%$ CI $0.88-1.07$ & $828(4.75)$ \\
\hline Myocardial infarction (rate ${ }^{b}$ ) & $267(1.46)$ & HR $1.04,95 \%$ CI $0.88-1.24$ & $257(1.39)$ \\
\hline Refractory angina $\left(\right.$ rate $\left.^{b}\right)$ & $150(0.81)$ & HR $0.86,95 \%$ CI $0.69-1.07$ & $174(0.94)$ \\
\hline New overt heart failure (rate ${ }^{\mathrm{b}}$ ) & $86(0.46)$ & HR $0.71,95 \%$ CI $0.54-0.94$ & $121(0.65)$ \\
\hline Debilitating stroke (rate $\left.{ }^{b}\right)$ & $77(0.41)$ & HR $0.78,95 \%$ CI $0.58-1.05$ & $99(0.53)$ \\
\hline Any stroke or TIA ${ }^{\mathrm{e}}\left(\right.$ rate $\left.^{\mathrm{b}}\right)$ & $187(1.01)$ & HR $0.72,95 \%$ CI $0.60-0.87$ & $258(1.40)$ \\
\hline Peripheral revascularisation $\left(\right.$ rate $^{b}$ ) & $146(0.79)$ & HR $1.25,95 \%$ CI $0.98-1.59$ & $118(0.63)$ \\
\hline Coronary angiography (rate $\left.{ }^{\mathrm{b}}\right)$ & $895(5.46)$ & HR $0.82,95 \%$ CI $0.75-0.90$ & $1068(6.69)$ \\
\hline Percutaneous coronary intervention (rate ${ }^{b}$ ) & $385(2.15)$ & HR $0.92,95 \%$ CI $0.80-1.06$ & $417(2.34)$ \\
\hline Coronary bypass surgery (rate ${ }^{\mathrm{b}}$ ) & $294(1.62)$ & HR $0.79,95 \%$ CI $0.68-0.92$ & $371(2.06)$ \\
\hline Death, CV events or procedures (rate ${ }^{\mathrm{b}}$ ) & $1439(9.32)$ & HR $0.89,95 \%$ CI $0.83-0.95$ & $1583(10.50)$ \\
\hline
\end{tabular}

Note. SD denotes standard deviation, MI myocardial infarction, CAD coronary artery disease, NYHA New York Heart Association, CV cardiovascular, BP blood pressure (cuff method), TIA transient ischemic attacks, HR hazard ratio, CI confidence interval.

aStroke, transient ischemic attacks or claudication.

${ }^{b}$ Number of patients with (combined) event per 100 patient-years at risk.

${ }^{\mathrm{c}}$ Death of any cause, myocardial infarction or debilitating stroke.

${ }^{\mathrm{d}}$ Death of any cause, myocardial infarction, refractory angina, new overt heart failure, debilitating stroke or peripheral revascularisation.

${ }^{\mathrm{e}}$ Includes non-confirmed strokes diagnosed by investigators and transient ischemic attacks.

of gastrointestinal bleeding between the two treatment groups. In the COSTART body system musculoskeletal, leg cramps occurred significantly more frequently in patients assigned nifedipine. In the COSTART body system nervous, dizziness was reported frequently (5/100 patient-years) and with equal frequency in both treatment groups. Depression, cerebral ischaemia, amnesia, cerebral haemorrhage, and dysarthria were all significantly less often reported in patients assigned nifedipine. In the COSTART body system respiratory, respiratory disorder and hyperventilation were more frequently reported for patients assigned nifedipine 


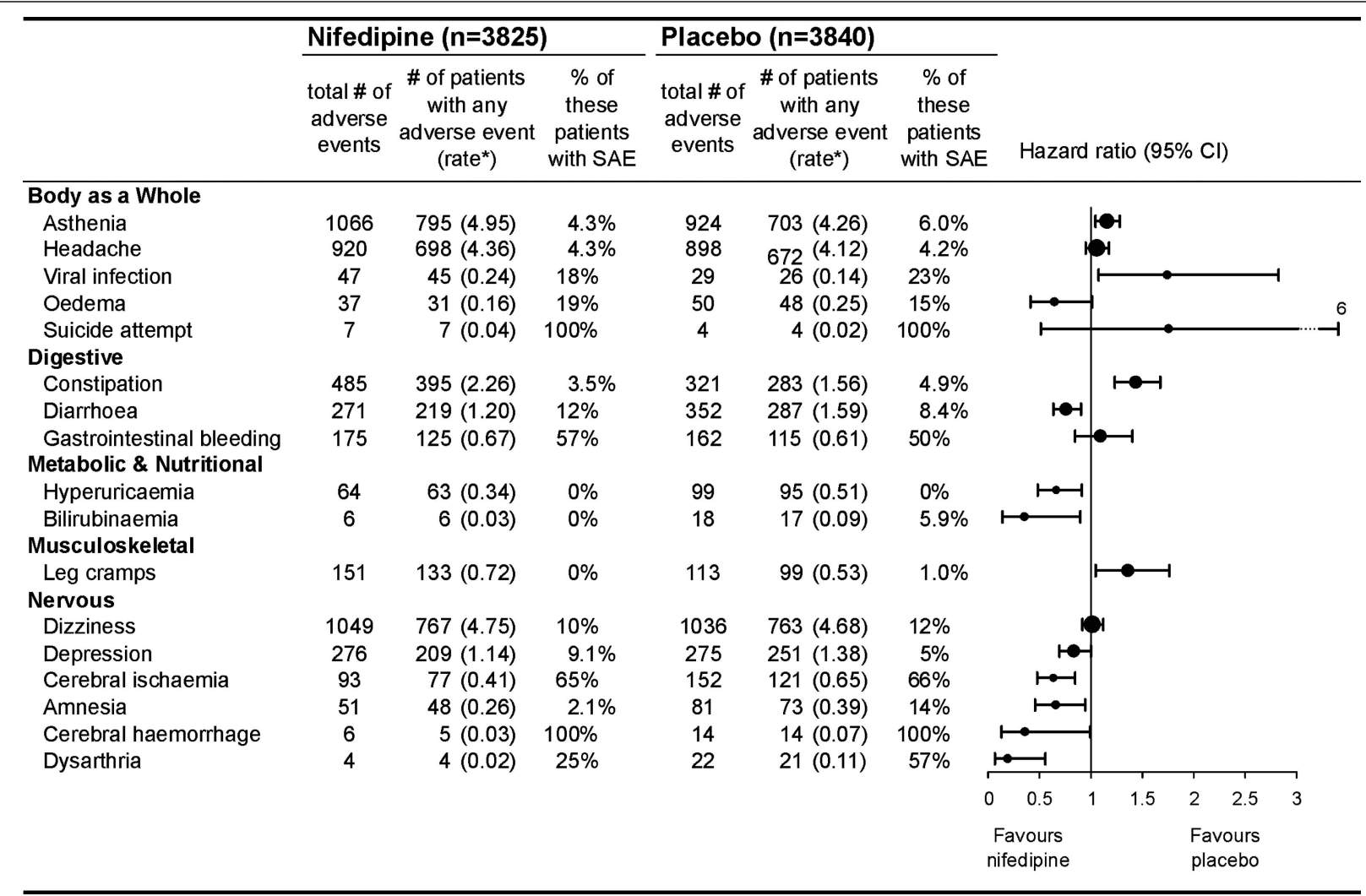

Fig. 3. Break-down of adverse events by COSTART body system class. Note: Dot size indicating the hazard ratio corresponds to the percentage of patients with the event concerned <3\% (smallest dot), 3-15\% (medium-size dot), >15\% (largest size dot). SAE denotes serious adverse event and CI confidence interval. ${ }^{*}$ Number of patients with event per 100 patient-years at risk.

whilst coughing and apnoea occurred more frequently in the placebo group. In the COSTART body system skin and appendages, there were significant differences for eczema in favour of placebo and sweating in favour of nifedipine. Urinary tract infection, impotence, polyuria, urinary tract disorder and testis disorder were associated with nifedipine, while vaginitis and gynaecomastia were associated with placebo.

The numbers of patients in each treatment group for whom the presence of any form of cancer was known at entry into the trial were similar: 170 (4.4\%) nifedipine, $186(4.8 \%)$ placebo. The numbers of patients without a baseline history of cancer who were diagnosed with cancer during follow-up are shown in Figure 5. Death or the development of any cancer in patients without a baseline cancer history occurred in 502 and 454 patients assigned nifedipine and placebo respectively. The corresponding event rates were 2.88/100 patient-years for nifedipine and 2.58/100 patient-years for placebo (HR $1.11,95 \%$ CI 0.98-1.26). Any type of cancer was reported for 311 patients assigned nifedipine and 262 patients assigned placebo. The excess of 49 cases of any type of cancer ( 0.29 per 100 years of follow-up) in the nifedipine group was attributable almost entirely to an excess of cancer within the COSTART body systems skin and appendages (24 excess cases) and urogenital (27 excess cases) in patients assigned nifedipine. Basal/squamous cell carcinoma occurred in 63 patients assigned nifedipine and 43 patients assigned placebo. Five patients with non-melanoma skin cancer died during the study. None of these deaths were related to the skin cancer. Excluding non-melanoma skin cancer, death or cancer occurred in 445 patients assigned nifedipine and 419 assigned placebo corresponding to rates of $2.53 / 100$ patient years and 2.37/100 patient years (HR 1.06, 95\% CI 0.93-1.22).

The occurrence of cancer in the urogenital system was more marked in females than in males. The total number of women who had any urogenital cancer was 25 among those assigned nifedipine and eight among placebo. The corresponding event rates were 0.70 and 0.22 per 100 patient years respectively (HR 3.24, 95\% CI 1.46-7.17). Breast cancer was diagnosed in 15 female patients assigned nifedipine and seven female patients assigned placebo (0.42/100 patient years and 0.19/100 patient years for female patients allocated nifedipine and placebo respectively, rate difference $0.23 / 100 \mathrm{pa}-$ tient years). Breast cancer was also diagnosed in two male patients-one each in the nifedipine and placebo treatment groups. In Figure 5, other urogenital cancers included endometrial cancer in five patients as- 


\begin{tabular}{|c|c|c|c|c|c|c|c|c|c|c|c|}
\hline & \multicolumn{3}{|c|}{ Nifedipine $(n=3825)$} & \multicolumn{3}{|c|}{ Placebo $(n=3840)$} & \multirow{2}{*}{\multicolumn{5}{|c|}{ Hazard ratio $(95 \% \mathrm{Cl})$}} \\
\hline & $\begin{array}{c}\text { total \# of } \\
\text { adverse } \\
\text { events }\end{array}$ & $\begin{array}{l}\text { \# of patients } \\
\text { with any } \\
\text { adverse event } \\
\left(\text { rate }^{*}\right)\end{array}$ & $\begin{array}{c}\% \text { of } \\
\text { these } \\
\text { patients } \\
\text { with SAE }\end{array}$ & $\begin{array}{l}\text { total \# of } \\
\text { adverse } \\
\text { events }\end{array}$ & $\begin{array}{l}\text { \# of patients } \\
\text { with any } \\
\text { adverse event } \\
\left(\text { rate }^{*}\right)\end{array}$ & $\begin{array}{c}\text { \% of } \\
\text { these } \\
\text { patients } \\
\text { with SAE }\end{array}$ & & & & & \\
\hline \multicolumn{12}{|l|}{ Respiratory } \\
\hline Cough increased & 457 & $371(2.08)$ & $4.0 \%$ & 511 & $427(2.40)$ & $2.8 \%$ & n & & & & \\
\hline Respiratory disorder & 56 & $52(0.28)$ & $31 \%$ & 39 & $34(0.18)$ & $35 \%$ & & $\rightarrow$ & & & 16 \\
\hline Hyperventilation & 14 & $14(0.07)$ & $29 \%$ & 4 & $3(0.02)$ & $33 \%$ & & & & $\bullet-\cdots .$. & \\
\hline Apnoea & 13 & $13(0.07)$ & $100 \%$ & 27 & $26(0.14)$ & $88 \%$ & 10 & & & & \\
\hline \multicolumn{11}{|l|}{ Skin and Appendages } & \\
\hline Eczema & 136 & $108(0.58)$ & $0 \%$ & 87 & $76(0.40)$ & $0 \%$ & & $\mapsto$ & & & \\
\hline Sweating & 103 & $90(0.48)$ & $7.8 \%$ & 157 & $135(0.73)$ & $6.7 \%$ & 101 & & & & \\
\hline \multicolumn{12}{|l|}{ Urogenital } \\
\hline Urinary tract infection & 298 & $214(1.17)$ & $24 \%$ & 220 & $164(0.88)$ & $20 \%$ & & •-1 & & & \\
\hline Impotence & 134 & $123(0.67)$ & $0.8 \%$ & 92 & $85(0.46)$ & $0 \%$ & & $\mapsto-1$ & & & \\
\hline Polyuria & 38 & $36(0.19)$ & $2.8 \%$ & 20 & $20(0.11)$ & $0 \%$ & & 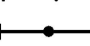 & -1 & & \\
\hline Urinary tract disorder & 39 & $33(0.18)$ & $30 \%$ & 18 & $17(0.09)$ & $29 \%$ & & & & & \\
\hline Testis disorder & 31 & $27(0.14)$ & $19 \%$ & 17 & $14(0.07)$ & $21 \%$ & & & $\longrightarrow$ & & \\
\hline Vaginitis & 9 & $9(0.05)$ & $0 \%$ & 22 & $20(0.11)$ & $0 \%$ & ro- & & & & \\
\hline \multirow[t]{3}{*}{ Gynaecomastia } & 6 & $6(0.03)$ & $0 \%$ & 20 & $18(0.10)$ & $0 \%$ & $10-1$ & & & & \\
\hline & & & & & & & 0 & 2 & 3 & 5 & \\
\hline & & & & & & & $\begin{array}{l}\text { Favours } \\
\text { nifedipine }\end{array}$ & & $\begin{array}{l}\text { Favours } \\
\text { placebo }\end{array}$ & & \\
\hline
\end{tabular}

Fig. 4. Break-down of adverse events by COSTART body system class. Note: Dot size indicating the hazard ratio corresponds to the percentage of patients with the event concerned <3\% (smallest dot), 3-15\% (medium-size dot), >15\% (largest size dot). SAE denotes serious adverse event and CI confidence interval. ${ }^{*}$ Number of patients with event per 100 patient-years at risk.

signed nifedipine and one patient assigned placebo, ovarian cancer in four patients assigned nifedipine and no patient assigned placebo, and cervical cancer in one patient assigned nifedipine and no patient assigned placebo.

\section{Discussion}

In the 1990s there was much controversy concerning the safety of calcium channel antagonists focussing on short acting preparations and clinical outcomes relating to myocardial infarction, gastrointestinal haemorrhage, suicide and cancer. The ACTION study was a large and long randomised trial using the long-acting preparation of the calcium channel antagonist nifedipine in patients with stable symptomatic angina pectoris. Its main results are summarised in Table 1.

ACTION provided an opportunity for a detailed examination of adverse events. For the present purpose we have analysed the complete ACTION safety database, but report only COSTART codeable adverse events considered relevant (c.f. statistical methods). It follows that there were no differences between treatment groups for events not mentioned. Screening the safety data base for differences and selecting events for inclusion in the present report involved multiple testing of statistical significance. We emphasise that this has implications for cumulative type I errors and increases the probability of chance findings. The more conservative approach is to adjust for multiple testing when calculating $p$-values and confidence intervals. This increases the probability that legitimately significant results will not be detected because of a type II error. Hence, we have opted to use unadjusted tests, and report hazard ratios with $95 \%$ confidence intervals rather than $p$-values. As always, the findings should be considered in relation to plausibility, existing scientific knowledge, specificity and coherence. Furthermore, in interpreting our findings it is imperative to consider the width of the confidence intervals reported. A wide confidence interval for a rare event indicates that our data does not allow a definitive conclusion even when the interval does not include unity for the hazard ratio.

The present analysis corroborates our previously published results. The finding that nifedipine reduced the incidence of new overt heart failure as classified by the ACTION Critical Events Committee was novel [29]. The latter was based on strict criteria. A similar conclusion with respect to heart failure was obtained based on adverse events as reported by investigators. In addition, nifedipine reduced the rate of angina pectoris, hypertension, bradycardia, and 


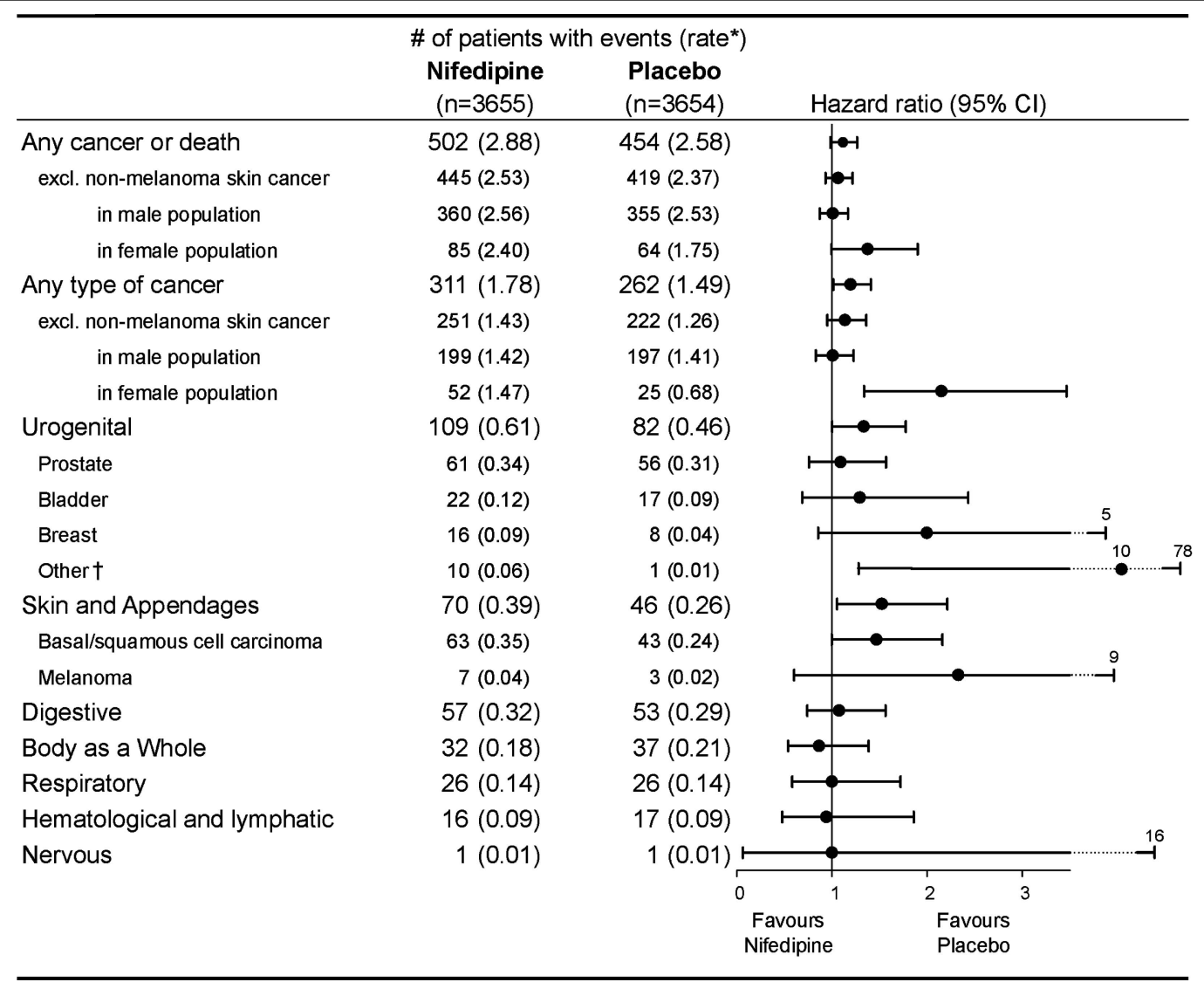

Fig. 5. Occurrence of cancer among patients without cancer at baseline. Note: CI denotes confidence interval. ${ }^{*}$ Number of patients with event per 100 patient-years at risk. ${ }^{\dagger}$ Includes ovarian, endometrial and cervix carcinoma.

cerebral ischemia and haemorrhage. As expected, peripheral oedema in the absence of heart failure was more common with nifedipine. The MATH trial also reported that peripheral oedema was among the most common adverse events of nifedipine GITS, but that the oedema was not associated with overall body fluid retention [36].

The known side effects of nifedipine, including peripheral oedema, vasodilatation, hypotension, asthenia, constipation, leg cramps, non-specific respiratory complaints, impotence and polyuria were reported more frequently for patients assigned nifedipine (Figure 2). The incidence rates of most other reported adverse events were similar. There was an increase in the rate of viral infection and urinary tract infection in the nifedipine group but the rate difference between the two groups was small $(0.10 / 100$ patient years and $0.28 / 100$ patient years for viral infection and urinary tract infection respectively). There was no increase in the occurrence of myocardial infarction or suicide. Major bleeding is another alleged adverse event with calcium channel antagonists. Current evidence is contradictory, and data specific to nifedipine is scarce [23]. The findings in ACTION show that nifedipine GITS does not cause major gastrointestinal haemorrhage.

Many different types of cardiovascular drugs, including antihypertensives, statins and angiotension receptor blockers have been reported to increase the risk of cancer [37-39]. A recent review of current evidence concluded that although there may be a weak association of $\beta$-blockers and diuretics therapy with malignancy, the management of hypertension following current treatment guidelines can be continued with little fear of any substantial risk of cancer [40]. Data about the risk of cancer in calcium channel antagonists users are controversial $[23,40]$. Observational studies suggested that use of calcium channel antagonists could be associated with increased risk of malignancy [16,17]. Three large case-controlled studies showed no association [41-43]. In a recent cohort study, where detailed information on the drug use was available and potential confounders were well controlled, an increased risk of cancer was reported with verapamil, but the risk was not associated with diltiazem or nifedipine use [44].

In the present study the rate of death or any cancer (including non-melanoma skin cancer) was slightly higher but not statistically different between the two 
treatment groups. The data on particular cancers is based on small numbers of cases and significant differences could represent chance findings. The imbalance in cancer between the two groups is driven by nonmelanoma skin cancers, which are usually excluded from evaluations [32,33], and by urogenital cancers in females. The number of females in the nifedipine and placebo groups were 784 and 797 . The small number of women limits the reliability of any statistical assessment.

The adverse and serious adverse events reported in the ACTION trial demonstrate that nifedipine GITS is a well tolerated drug for use in the treatment of angina with clear clinical benefit on cardiovascular events and the rate of procedures. The information reported allows the physician to balance efficacy and safety, and provide robust information to patients on side-effects of the drug.

\section{Appendix}

For combined clinical entities shown in Figures 2 and 3 , the COSTART terms included are listed below (COSTART code between brackets).

Bradycardia: bradycardia (02010150), sinus bradycardia (02010500).

Tachycardia: tachycardia (02010570), supraventricular tachycardia (02010540), ventricular tachycardia (02010630), nodal tachycardia (02010400).

Hypotension: hypotension (02030270), postural hypotension (02030470).

Heart failure: heart failure (02010310), congestive heart failure (02010180), cardiomegaly (02010170), left heart failure (02010340), cardiomyopathy (02010175), venous pressure increased (02030730), pulmonary oedema (09050120), lung disorder (09050110) when preferred term contains congestion or rales unless the investigator terms contains sounds. Included are also the investigator terms fluid in lungs or worsening pulmonary stasis.

Other electrocardiogram abnormality: electrocardiogram abnormal (02010260), ST depressed (02010510), $\mathrm{T}$ inverted (02010560), QT interval prolonged (02010480), ST elevated (02010520), T amplitude decreased (02010550),

Conduction abnormalities: AV block complete (02010100), AV block first degree (02010110), AV block 2nd degree (02010120), AV block (02010130), Adams stokes syndrome (02010010), heart block (02010300), PR interval prolonged (02010450), bundle branch block (02010160)

Premature complexes: ventricular arrhythmia (02010590), ventricular extrasystoles (02010600), extrasystoles (02010280), bigeminy (02010140), ectopia cordis (02010250).

Cardiac arrest: heart arrest (02010290), ventricular fibrillation (02010610).

Atrial arrhythmia: atrial arrhythmia (02010060), atrial flutter (02010080), atrial fibrillation (02010070), nodal arrhythmia (02010390), arrhythmia (02010050), supraventricular extrasystoles (02010530).

Angina pectoris: angina pectoris (02010030), myocardial ischaemia (02030380), coronary occlusion (02010220), coronary thrombosis $(02010230)$.

Thrombophlebitis: thrombophlebitis (02030600), phlebitis (02030450), deep thrombophlebitis (02030200).

Peripheral vascular disorder: vascular disorder (02030660), peripheral vascular disorder (02030440), occlusion (02030400), carotid occlusion (02030080), arteriosclerosis (02030040), peripheral gangrene (02030430).

Gastrointestinal bleeding: gastrointestinal hemorrhage (03090100), melena (03090140), hematemesis (03090120), hemorrhagic gastritis (03160050), stomach ulcer hemorrhage (03160100), duodenal ulcer hemorrhage (03040010), esophageal hemorrhage (03060030), hemorrhage of colon (03030020), peptic ulcer hemorrhage (03080010).

\section{Acknowledgements}

We thank all the patients who participated in this trial and all the ACTION investigators and study nurses/coordinators whose work made the trial possible. The study was supported by Bayer Healthcare AG, Wuppertal, Germany.

\section{References}

1. Lenfant C. The calcium channel blocker scare. Lessons for the future. Circulation 1995;91:2855-2856.

2. Opie LH, Messerli FH. Nifedipine and mortality. Grave defects in the dossier. Circulation 1995;92:10681073.

3. Opie LH. Risks and benefits of calcium antagonists. Lancet 1995;346:961.

4. Horton R. Spinning the risks and benefits of calcium antagonists. Lancet 1995;346:586-587.

5. Poole-Wilson PA. The calcium antagonist controversy; implications beyond drug prescription. Eur Heart J 1996;17:11311133.

6. Kaplan NM. Do calcium antagonists cause myocardial infarction? Am J Cardiol 1996;77:81-82.

7. Kaplan NM. Do calcium antagonists cause death, gastrointestinal bleeding, and cancer? Am J Cardiol 1996;78:932-933.

8. McMurray J, Murdoch D. Calcium-antagonist controversy: The long and short of it? Lancet 1997;349:585-586.

9. Califf RM, Kramer JM. What have we learned from the calcium channel blocker controversy? Circulation 1998;97:1529-1531.

10. Lubsen J. The calcium channel antagonist debate: Recent developments. Eur Heart J 1998;19(Suppl I):I3-I7.

11. Stelfox HT, Chua G, O'Rourke K, Detsky AS. Conflict of interest in the debate over calcium-channel antagonists. $N$ Engl J Med 1998;338:101-106.

12. Psaty BM, Heckbert SR, Koepsell TD, et al. The risk of myocardial infarction associated with antihypertensive drug therapies. JAMA 1995;274:620-625. 
13. Furberg CD, Psaty BM, Meyer JV. Nifedipine. Dose-related increase in mortality in patients with coronary heart disease. Circulation 1995;92:1326-1331.

14. Pahor M, Guralnik JM, Corti MC, Foley DJ, Carbonin P, Havlik RJ. Long-term survival and use of antihypertensive medications in older persons. J Am Geriatr Soc 1995;43:1191-1197.

15. Furberg CD, Psaty BM. Should calcium antagonists be firstline agents in the treatment of cardiovascular disease? The public health perspective. Cardiovasc Drugs Ther 1996;10:463466.

16. Pahor M, Guralnik JM, Ferrucci L, et al. Calcium-channel blockade and incidence of cancer in aged populations. Lancet 1996;348:493-497.

17. Pahor M, Guralnik JM, Salive ME, Corti MC, Carbonin P, Havlik RJ. Do calcium channel blockers increase the risk of cancer? Am J Hypertens 1996;9:695-699.

18. Pahor M, Guralnik JM, Furberg CD, Carbonin P, Havlik R. Risk of gastrointestinal haemorrhage with calcium antagonists in hypertensive persons over 67 years old. Lancet 1996;347:1061-1065.

19. Garcia Rodriguez LA, Cattaruzzi C, Troncon MG, Agostinis L. Risk of hospitalization for upper gastrointestinal tract bleeding associated with ketorolac, other nonsteroidal anti-inflammatory drugs, calcium antagonists, and other antihypertensive drugs. Arch Intern Med 1998;158:33-39.

20. Kaplan RC, Heckbert SR, Koepsell TD, Rosendaal FR, Psaty BM. Use of calcium channel blockers and risk of hospitalized gastrointestinal tract bleeding. Arch Intern Med 2000;160:1849-1855.

21. Lindberg G, Bingefors K, Ranstam J, Rastam L, Melander A. Use of calcium channel blockers and risk of suicide: Ecological findings confirmed in population based cohort study. BMJ 1998;316:741-745.

22. Opie LH, Yusuf S, Kubler W. Current status of safety and efficacy of calcium channel blockers in cardiovascular diseases: A critical analysis based on 100 studies. Prog Cardiovasc Dis 2000;43:171-196.

23. Kizer JR, Kimmel SE. Epidemiologic review of the calcium channel blocker drugs. An up-to-date perspective on the proposed hazards. Arch Intern Med 2001;161:1145-1158.

24. Eisenberg MJ, Brox A, Bestawros AN. Calcium channel blockers: An update. Am J Med 2004;116:35-43.

25. Pitt B, Byington RP, Furberg CD, et al. Effect of amlodipine on the progression of atherosclerosis and the occurrence of clinical events. PREVENT Investigators. Circulation 2000;102:1503-1510.

26. Rehnqvist N, Hjemdahl P, Billing E, et al. Effects of metoprolol vs verapamil in patients with stable angina pectoris. The Angina Prognosis Study in Stockholm (APSIS). Eur Heart $J$ 1996;17:76-81.

27. Fox KM, Mulcahy D, Findlay I, Ford I, Dargie HJ. The Total Ischaemic Burden European Trial (TIBET). Effects of atenolol, nifedipine SR and their combination on the exercise test and the total ischaemic burden in 608 patients with stable angina. The TIBET Study Group. Eur Heart J 1996;17:96-103.

28. Dargie HJ, Ford I, Fox KM. Total Ischaemic Burden European Trial (TIBET). Effects of ischaemia and treatment with atenolol, nifedipine SR and their combination on outcome in patients with chronic stable angina. The TIBET Study Group. Eur Heart J 1996;17:104-112.
29. Poole-Wilson PA, Lubsen J, Kirwan BA, et al. Effect of longacting nifedipine on mortality and cardiovascular morbidity in patients with stable angina requiring treatment (ACTION trial): Randomised controlled trial. Lancet 2004;364:849-857.

30. Lubsen J, Poole-Wilson PA, Pocock SJ, et al. Design and current status of ACTION: A Coronary disease Trial Investigating Outcome with Nifedipine GITS. Gastro-Intestinal Therapeutic System. Eur Heart J 1998;19(Suppl I):I20-I32.

31. COSTART. In: Rockville MD (Eds.), Coding Symbols for Thesaurus of Adverse Reaction Terms, 5th ed. Food and Drug Administration, Center for Drug Evaluation and Research, 1995.

32. MRC/BHF. Heart Protection Study of cholesterol lowering with simvastatin in 20,536 high-risk individuals: A randomised placebo-controlled trial. Lancet 2002;360:7-22.

33. Randomised trial of cholesterol lowering in 4444 patients with coronary heart disease: The Scandinavian Simvastatin Survival Study (4S). Lancet 1994;344:1383-1389.

34. Frison LJ, Pocock SJ. Linearly divergent treatment effects in clinical trials with repeated measures: Efficient analysis using summary statistics. Stat Med 1997;16: 2855-2872.

35. Lubsen J, Wagener G, Kirwan BA, de Brouwer S, PooleWilson PA. Effect of long-acting nifedipine on mortality and cardiovascular morbidity in patients with symptomatic stable angina and hypertension: The ACTION trial. $J$ Hypertens 2005;23:641-648.

36. Krakoff LR. Effectiveness of nifedipine gastrointestinal therapeutic system for treatment of hypertension: Results of the MATH Trial. $J$ Cardiovasc Pharmacol 1993;21(Suppl 2):S14-S17.

37. Pfeffer MA, Swedberg K, Granger CB, et al. Effects of candesartan on mortality and morbidity in patients with chronic heart failure: The CHARM-Overall programme. Lancet 2003;362:759-766.

38. Shepherd J, Blauw GJ, Murphy MB, et al. Pravastatin in elderly individuals at risk of vascular disease (PROSPER): A randomised controlled trial. Lancet 2002;360: 1623-1630.

39. Sacks FM, Pfeffer MA, Moye LA, et al. The effect of pravastatin on coronary events after myocardial infarction in patients with average cholesterol levels. Cholesterol and Recurrent Events Trial investigators. N Engl J Med 1996;335:1001-1009.

40. Grossman E, Messerli FH, Goldbourt U. Carcinogenicity of antihypertensive therapy. Curr Hypertens Rep 2002;4:195-201.

41. Jick H, Jick S, Derby LE, Vasilakis C, Myers MW, Meier CR. Calcium-channel blockers and risk of cancer. Lancet 1997;349:525-528.

42. Rosenberg L, Rao RS, Palmer JR, et al. Calcium channel blockers and the risk of cancer. JAMA 1998;279:10001004 .

43. Stahl M, Bulpitt CJ, Palmer AJ, Beevers DG, Coles EC, Webster J. Calcium channel blockers, ACE inhibitors, and the risk of cancer in hypertensive patients: A report from the Department of Health Hypertension Care Computing Project (DHCCP). J Hum Hypertens 2000;14:299304.

44. Beiderbeck-Noll AB, Sturkenboom MC, van der Linden $\mathrm{PD}$, et al. Verapamil is associated with an increased risk of cancer in the elderly: The Rotterdam study. Eur $J$ Cancer 2003;39:98-105. 